Traversées québécoises en littérature féministe : influences états-uniennes et françaises

\title{
Aurore Turbiau
}

Klincksieck | «Revue de littérature comparée » 2020/3 n 375 | pages 297 à 316 ISSN 00351466 ISBN 9782252045053 Article disponible en ligne à l'adresse : https://www.cairn.info/ revue-de-litterature-comparee-2020-3-page-297.htm

\section{Version de pré-publication}

Cet article se penchera sur les collaborations qui ont eu lieu entre le Québec, les ÉtatsUnis et la France dans le mouvement féministe tel qu'il s'est construit littérairement pendant la décennie 1970, pleine période des luttes de libération des femmes dans ces trois pays. Le Québec est un lieu d'intersection : lié par son histoire culturelle à la France, par sa géographie aux États-Unis, divisé entre l'appartenance à l'entité fédérale canadienne et le rejet de l'ex-colonialisme anglais. Or le féminisme, culture à part à l'intérieur de la culture québécoise, favorise particulièrement les rapports avec la France et les États-Unis, « centres ${ }^{1}$ » hégémoniques des théories féministes et littéraires occidentales à l'époque. Le moment du féminisme est en effet pour bien des écrivaines québécoises le moment où elles renforcent leurs liens avec les Françaises ; en même temps il s'agit d'une forme de contre-culture et le rapprochement avec les États-Unis, pionniers en matière de féminisme, apparaît indispensable. J'étudierai la manière dont ces liens s'établissent et la manière dont la littérature féministe québécoise se construit ainsi à travers des dialogues internationaux.Quelques notions doivent être définies d'entrée de jeu afin que les rapports d'influence soient bien compris. Je parle de « littérature féministe » : j'entends par là, de manière large, la littérature qui est produite dans « l'espace de la cause des femmes ${ }^{2} »$. C'est une littérature qui porte des enjeux politiques concernant les

1. Voir Pascale Casanova, La République mondiale des lettres, Paris, Éditions du Seuil, 1999.

2. Voir Laure Bereni, «Penser la transversalité des mobilisations féministes : l'espace de la cause des femmes », dans Christine Bard (dir.), Les Féministes de la deuxième vague, Rennes, Presses Universitaires de Rennes, 2012, p. 27-42. 
A. Turbiau. Traversées québécoises en littérature féministe

femmes et qui recherche l'amélioration de leur condition ; selon les écrivaines des différents pays, ce projet peut prendre des formes différentes. Certaines autrices tendent au matérialisme : elles considèrent que la lutte doit consister à mettre en avant les structures sociales et économiques qui créent l'exploitation des femmes ; elles ont un point de vue «constructiviste » dans la mesure où elles considèrent que la condition féminine est une construction culturelle non fondée en nature. Le matérialisme étant un héritage du marxisme, on parle de rapport entre «base » et «superstructure », d'exploitation économique, de « classes sociales » et de «classes de sexe », d'appropriation des moyens de production — et de patriarcat puisqu'il s'agit de féminisme ${ }^{1}$. Le même courant de pensée prend des noms différents selon les contextes : en France on parle au début de la décennie plutôt de « féministes révolutionnaires », puis la désignation «féministes matérialistes » prend le relais et permet de marquer plus de distance avec le marxisme; aux États-Unis et au Québec on parle volontiers, pour nommer des théories proches et centrées sur l'idée de l'autonomie des luttes féministes, de «féministes radicales ». En France, pendant la décennie 1970, Monique Wittig, Françoise d'Eaubonne, Christine Delphy et quelques autres incarnent ce courant ; aux États-Unis, ce sont essentiellement Ti-Grace Atkinson, Kate Millett, Shulamith Firestone, Susan Brownmiller. En littérature française, la veine matérialiste se traduit de diverses manières ${ }^{2}$ : d'abord, par une forte disproportion entre la production essayistique - assez grande - et la production fictionnelle ou poétique - assez pauvre en nombre. Certaines écrivaines préfèrent renoncer en partie à l'aspect littéraire de leur écriture pour se concentrer, dans certains livres, sur la charge polémique - c'est le cas de Christiane Rochefort ou Françoise d'Eaubonne pour certains de leurs ouvrages ${ }^{3}$; d'autres - Monique Wittig au premier chef — chargent leur œuvre et le travail de la langue qui s'y joue de devenir facteurs de révolution, distinguant leur lutte hors lit-

1. Voir dans Delphy, l'article « Lennemi principal » qui pose pour la première fois pour les Françaises et pour les Québécoises les bases d'une analyse matérialiste de la société patriarcale. Christine Delphy, L'Ennemi principal. Économie politique du patriarcat, Paris, Éditions Syllepse, [1970] 2017.

2. On retrouve à peu de choses près les mêmes partages idéologiques entre les féminismes français et les féminismes québécois pendant les années 1970, notamment parce que les mêmes textes circulent entre la France et le Québec. Il était important ici d'expliquer plus particulièrement le développement des idées matérialistes, qui se sont d'abord développées sur le sol français, pour la suite de l'analyse. Mais au Québec, Les Têtes de pioche par exemple (revue représentative d'une part du féminisme québécois à partir de 1976) se sont plutôt positionnées comme «radicales»: inspirées par les idées de la gauche et du marxisme, mais autonomes dans leur féminisme - elles ne se réfèrent pas explicitement aux théories dites matérialistes, quoique on en retrouve les analyses dans leurs textes.

3. Citons par exemple Les Enfants d'abord de Christiane Rochefort (1976), qui associe la lutte pour les droits des enfants à celle pour les droits des femmes ; Histoire et actualité du féminisme ou Le Féminisme ou la mort (1972, 1974) de Françoise d'Eaubonne. Nous pouvons sans doute ajouter à cette liste Ainsi soit-elle de Benoîte Groult (1975), avec une réserve : Benoîte Groult était moins directement impliquée dans le mouvement des femmes et les luttes sociales des années 1970 que Françoise d'Eaubonne ou Christiane Rochefort. 
A. Turbiau. Traversées québécoises en littérature féministe

térature, active et revendicatrice, de leur lutte proprement littéraire, hors polémique et plus orientée vers un travail formel — «matérialiste » également mais dans le sens d'un travail précis sur la langue. Sur le curseur des positions que j'appelle ici « féministes », on trouve aussi des écrivaines qui, sans forcément rejeter radicalement les analyses féministes matérialistes, ne leur donnent pas place dans leur œuvre et préfèrent se consacrer à la recherche d'une nouvelle culture «féminine »: elles se placent en quelque sorte au-delà de la révélation des rouages du patriarcat et souhaitent entraîner une révolution presque seulement par le travail du langage, considérant que les femmes, en tant que femmes, ont des choses nouvelles à ap porter à la culture. On parle alors du mouvement de l'« écriture féminine »; si la démarche d'écriture qui y est liée ressemble, sur bien des points, à celle d'une Monique Wittig, elle s'en détache par son substrat politique - dans un cas, la révolution souhaitée ne remet pas complètement en cause les grandes catégories sexuelles mais les retravaille (écriture féminine), dans l'autre l'écriture doit idéalement amener un bouleversement de tout le système hétéropatriarcal (matérialisme de Wittig). Hélène Cixous parle de l'écriture féminine en ces mots, en 1975 dans La Jeune Née :

D’une certaine manière l'écriture féminine ne cesse de résonner du déchirement qu'est pour la femme la prise de la parole orale — «prise » qui est effectuée comme un arrachement, un essor vertigineux et un lancer de soi, une plongée. Écoute parler une femme dans une assemblée (si elle n'a pas douloureusement perdu le souffle) : elle ne "parle » pas, elle lance dans l'air son corps tremblant, elle se lâche, elle vole, c'est toute entière qu'elle passe dans sa voix, c'est avec son corps qu'elle soutient vitalement la «logique » de son discours : sa chair dit vrai. Elle s'expose. En vérité, elle matérialise charnellement ce qu'elle pense, elle le signifie avec son corps. [...] Son discours, même " théorique » ou politique, n'est jamais simple ou linéaire, ou « objectivé », généralisé, elle entraîne dans l'histoire son histoire'.

Les principaux éléments de définition de l'« écriture féminine » sont ainsi posés : déchirement, métaphore, corps, oralité, non linéarité. Il faut remarquer que cette écriture a bien un rapport, à l'origine, avec la construction sociale qui fait des femmes des êtres humains voués à la discrétion et au silence : ainsi que l'explique Cixous, la prise de parole prend cette forme-là dans le contexte de l'« assemblée » où la femme est intimidée, contexte qui lui fait peur par la manière dont il est socialement construit — non habitué à voir parler une femme, la considérant d'abord par son corps. Si l'on traduit cette image en littérature, il s'agit du champ litté-

1. Hélène Cixous, Catherine Clément, La Jeune Née, Paris, Union générale d'édition, 1975, p. 170-171. 
A. Turbiau. Traversées québécoises en littérature féministe

raire ${ }^{1}$ dominé par les hommes et par un système de valeurs patriarcal, où la femme doit lutter pour imposer son écriture. Cela posé, il reste que l'« écriture féminine » de Cixous est un rapport au corps : la prise de parole «expose » la femme. Cette idée peut inciter certaines écrivaines à développer des visions « essentialistes », biologisantes; mais même si l'essentialisme est une tendance forte du mouvement de l'« écriture féminine », il faut se garder de systématiquement les associer - le corps peut aussi être compris comme une construction sociale nous verrons que les Québécoises, plus que les Françaises, lient souvent « écriture féminine » et analyse matérialiste de la société. Quelques théoriciennes incarnent particulièrement ce mouvement de « l'écriture féminine » : en France, ce sont tout particulièrement Hélène Cixous et Luce Irigaray; elles sont celles que l'on désigne lorsque, aux États-Unis, on parle du «French feminism ». Là-bas, Adrienne Rich et Mary Daly sont les principales représentantes d'un mouvement similaire de recherche d'une culture au féminin. Les tenantes françaises de l'«écriture féminine » refusent parfois d'être désignées comme «féministes ${ }^{2}$. Je les englobe toutefois dans le mouvement de la « littérature féministe » tel que je l'ai défini plus haut, pour les besoins de cet article : d'une part, avec leurs priorités propres, elles cherchent tout de même comme les féministes matérialistes à renverser la culture patriarcale, d'autre part leur influence a été très importante sur certaines écrivaines québécoises qui se revendiquent bien féministes.

Ces définitions posées, voyons maintenant quels rapports la littérature féministe québécoise entretient avec le féminisme international. Je montrerai d'abord que les Québécoises sont influencées dans leur pensée féministe par les États-uniennes, même si ce rapport est apparemment à sens unique et s'il ne va pas sans quelques frustrations. Nous verrons ensuite que les relations avec les Françaises sont beaucoup plus nourries malgré la persistance d'une certaine asymétrie ; les Québécoises construisent leur littérature féministe dans la continuité de celle qu'élaborent les Françaises, sans les suivre pour autant exactement sur les grands partages idéologiques qu'elles mettent en place dans leurs œuvres. Je propose de parler de « traversées québécoises » d'abord pour désigner ces voyages : le passage de la frontière nord-américaine d'un côté, de l'autre les explorations transatlantiques qui font se rencontrer personnes, œuvres et idées québécoises et françaises. Il ne s'agit pas vraiment d'échanges, à cause des asymétries qui demeurent dans les dialogues qui se nouent. Il ne s'agit pas non plus de simples

1. Pierre Bourdieu, Les Règles de l'art. Genèse et structure du champ littéraire, Paris, Éditions du Seuil, 1992.

2. C'est le cas d'Antoinette Fouque ou d'Hélène Cixous, qui affirme par exemple en 1977 que le féminisme est quasiment une force réactionnaire. Voir Hélène Cixous, « Entretien avec Françoise van Rossum-Guyon », Revue des sciences humaines, «Écriture, féminité, féminisme », n 168, 1977, p. 481. 
A. Turbiau. Traversées québécoises en littérature féministe

voyages, parenthèses temporelles, spatiales ou culturelles, qui offriraient aux Québécoises la possibilité d'un retour indemnes au pays. Il me semble qu'il y a bien plus traversées, carsi les Québécoises parcourent les cultures féministes et littéraires qui leur sont proches, celles des États-uniennes et des Françaises, elles s'en nourrissent sans s'y fixer. Elles passent à travers les mailles des hégémonies culturelles représentées par la France et les États-Unis, construisant une littérature féministe transverse par rapport à celles de ces pays, unique.

\section{Influences états-uniennes}

Si l'on s'intéresse aux rapports que les Québécoises entretiennent avec le monde anglophone, deux sphères culturelles différentes sont à considérer : celle des États-Unis et celle du Canada anglophone. Les liens sont distendus dans les deux cas mais ils ne sont pas de même nature : d'un côté les féministes québécoises s'inspirent de ce qui se passe dans le Women's lib états-unien et dans la contre-culture de manière plus générale, de l'autre les rapports avec le Canada anglophone sont tendus à cause de la question nationale québécoise. Même au sein d'une ville comme Montréal, les communautés française et anglaise entretiennent moins de rapports que l'on pourrait s'y attendre. Le fait est particulièrement frappant dans le cas des communautés féministes lesbiennes : les francophones ne se sont jamais vraiment intégrées aux communautés anglophones - ni l'inverse ${ }^{1}$. Pourtant les lesbiennes québécoises lisent, en anglais, les lesbiennes états-uniennes ; Diane Lamoureux explique même que ce sont majoritairement elles qui ont introduit les grands noms du féminisme américain dans les sphères féministes québécoises ${ }^{2}$. Le problème de la langue ne suffit donc pas à expliquer pourquoi féministes canadiennes anglophones et québécoises francophones n'échangent pas plus ; Gail Scott est en fait la seule écrivaine féministe québécoise de langue anglaise qui soit très régulièrement citée par ses consœurs de langue française. Dans cet article je me consacrerai donc plutôt aux rapports que les Québécoises entretiennent avec les États-uniennes, qui sont plus directement révélateurs de l'influence des théories anglophones sur leur écriture. Des collaborations nord-américaines entre littérature féministe québécoise et littérature féministe étatsunienne il faut surtout dire qu'elles sont peu nombreuses - mais elles existent. D'une manière générale, les rapports entre le Québec et les États-Unis ne sont pas très développés ; en ma-

1. Voir Andrea Hildebran, «Genèse d’une communauté lesbienne : un récit des années 1970 », dans Irène Demczuk, Frank W. Remiggi (dir.), Sortir de l'ombre. Histoires des communautés lesbienne et gaie de Montréal, Montréal, VLB éditeur, 1998, p. 207-234.

2. Diane Lamoureux, «La question lesbienne dans le féminisme montréalais : un chassé-croisé », dans Demczuk, Remiggi, op. cit., p. 167-196, p. 171. 
A. Turbiau. Traversées québécoises en littérature féministe

tière de littérature, les Québécois n'ont en général accès aux œuvres états-uniennes qu'une fois qu'elles ont été traduites en France ${ }^{1}$. Le cas de la littérature féministe, cependant, est un peu particulier : Paule Gilbert Lewis et Benoît Melançon expliquent que

Si l'on excepte la littérature féministe québécoise, on ne peut que constater l'absence de réels échanges entre les deux littératures ${ }^{2}$.

C'est justement par le féminisme qu'un contact est établi entre les deux sphères culturelles ; à lire les Québécoises on constate en effet qu'elles citent très souvent des autrices états-uniennes, surtout des théoriciennes ; c'est particulièrement vrai dans la littérature féministe lesbienne. En revanche, s'il est certain que les États-uniennes inspirent les Québécoises, difficile de dire si la réciproque a pu être vraie - a priori ce n'est pas le cas, ce qui est conforme par ailleurs au sort de la littérature québécoise aux États-Unis en général ${ }^{3}$; je vais donc surtout présenter ici ce que l'on sait des influences états-uniennes sur la pensée féministe québécoise.

Certaines des féministes québécoises voyagent aux États-Unis au cours des années 1970 ; le voyage aux États-Unis a pu être considéré comme un point de passage quasi obligé dans le parcours féministe - il en va d'ailleurs de même pour les féministes françaises ${ }^{4}$. En effet les États-uniennes ont apparemment une longueur d'avance : leur mouvement de libération a commencé entre 1963 et 1967, quelques années avant les premières coordinations d'ampleur des groupes féministes en France et au Québec. Aller lire et rencontrer les Étatsuniennes consiste donc à approcher les pionnières. Certains des livres de Nicole Brossard, tout particulièrement Amantes, retracent ces voyages. Certains lieux de voyage prennent en charge la signification même de ce contact nouveau avec l'étranger et avec le féminisme : le Barbizon par exemple, hôtel bien connu des étrangères et où Nicole Brossard loge lorsqu'elle est aux États-Unis — «Il y a toujours un hôtel dans ma vie pour me faire comprendre le patriarcat »,

1. Voir Paule Gilbert Lewis, «Literary Relationship between Quebec and the United States : A Meagre Reciprocity », dans Essays on Canadian Writing, n² 22, été 1981, p. 86-110.

2. Paule Gilbert Lewis, op. cit. Ici la citation est de Benoît Melançon, « La littérature québécoise et l'Amérique. Prolégomènes et bibliographie », Études françaises, volume 26, $\mathrm{n}^{\circ}$ 2, automne 1990, p. 71]: https://doi.org/10.7202/035817ar

3. Idem.

4. Dans un article intitulé «Lautre moitié de l'Amérique », Christiane Rochefort disait : «Je serai donc cette anomalie qui en 1973 n'est pas encore allée aux États-Unis. » — elle s'y rend finalement la même année pour un congrès d'écrivains. Citation tirée d'un texte dactylographié conservé dans les archives de Christiane Rochefort de l'IMEC, cote RCF 37.1 — reproduit ici avec l'accord de l'IMEC. À ce jour, je ne sais pas encore où l'article a été publié. 
A. Turbiau. Traversées québécoises en littérature féministe

dit-elle ${ }^{1}$. Elle rappelle fréquemment que c'est la rencontre avec la culture états-unienne qui a donné un nouveau tournant à son écriture et l'a lancée dans la littérature féministe :

Un livre a été écrit à l'époque où j'avais des préoccupations politiques en rapport avec toute la question nationale, alors que French kiss est arrivé à un moment où j'étais imbibée d'informations provenant de la contre-culture, d'informations qui touchaient la biologie, l'écologie, le corps dans des pratiques nouvelles [...] un autre lieu politique qui est celui $d u$ féminisme $e^{2}$.

Ce passage d'un état nationaliste de l'écriture à un état féministe a ainsi lieu entre 1974 et 1975, soit autour de son voyage américain. Si Nicole Brossard a été très attirée par les théories de Françaises comme Irigaray, Kristeva ou Wittig, elle explique en effet que ce sont tout de même les États-uniennes qui l'ont le plus influencée : «Je me sens beaucoup plus, au niveau des discours d'exploration théoriques, près d'elles, alors que les Françaises n'abordent le discours qu'à travers la psychanalyse ${ }^{3}$ ». Avec l'aide de Luce Guilbeault et de Margaret Wescott, elle tire de son voyage un film, intitulé Quelques féministes américaines, où elle mène des entretiens avec de grands noms du féminisme états-unien: Ti-Grace Atkinson, Rita Mae Brown, Kate Millett, Lila Karp, Betty Friedan, Margo Jefferson. Elle en parle dans son Journal intime :

New York, en 1975, quand Luce Guibeault et moi découvrions l'Amérique féministe et que nous sonnions à toutes les portes pour faire un bon film. Bouquet de fleurs à la main pour consoler Ti-Grace Atkinson de sa grippe, grand sourire et nervosité pour interviewer Kate Millett. Ou encore qu'avec un certain scepticisme nous attentions que l'ascenseur s'arrête au 24 e étage pour rencontrer Betty Friedan. [...] les sandwiches qui goûtent marxiste d'un côté et féministe de l'autre 4 .

Le film est projeté en 1977 à Montréal et à Chicago, il est ensuite proposé à la location à l'Office national du film du Canada : cela permet à celles qui ne voyagent pas de s'imprégner aussi de la culture féministe américaine. Au fil des années, Nicole Brossard cite de moins en moins ces féministes-là, et de plus en plus des écrivaines lesbiennes comme Mary Daly, Djuna

1. Nicole Brossard, Picture theory, Montréal, Éditions Nouvelle Optique, 1982,p. 30.

2. André Roy, « La fiction vive : entretien avec Nicole Brossard sur sa prose », dans John Robert Sorfleet (dir.), Les Romanciers québécois et leurs œuvres, Journal of Canadian Fiction, Guelph et Montréal, 1979, p. 31-40, p. 3536.

3. André Roy, op. cit., p. 39.

4. Nicole Brossard, Journal intime, Montréal, Édition Les Herbes rouges, 1984, p. 49. 
A. Turbiau. Traversées québécoises en littérature féministe

Barnes ou Adrienne Rich — qu'elle évoque même parfois par leur simple prénom, signifiant ainsi la proximité de leurs pensées ${ }^{1}$.

Dans des cas comme celui de Louky Bersianik, l'influence américaine est d'autant plus frappante qu'elle n'est pas, d'abord, de première main : dans L'Écriture, c'est les cris, elle explique en effet que, si c'est la découverte de Kate Millett qui lui a permis de se lancer dans l'écriture de L'Euguélionne ${ }^{2}$, elle l'a pourtant d'abord lue par le biais de compte rendus publiés dans la presse française ${ }^{3}$. Par la suite, son admiration pour la féministe américaine ne se dément pas, elle la cite à de très nombreuses reprises dans son œuvre, dans les dédicaces ou en notes de bas de page, voire de manière implicite par simple allusion à ses analyses politiques ${ }^{4}$. Bersianik cite souvent avec elle d'autres féministes influentes dont notamment, pour ne parler que des États-uniennes, Shulamith Firestone ${ }^{5}$. Par ailleurs, L'Euguélionne s'ouvre à New York l'Euguélionne arrive sur terre en criant «LIBERTÉ ! » et elle a « le poing levé vers le ciel de New York $^{6} »$ : cela peut signifier que les États-Unis représentent une terre d'accueil possible pour la révolution féministe ; cela peut signifier au contraire qu'ils sont un territoire où la lutte doit encore être menée. Il me semble que l'ambiguïté est significative car d'une manière générale les Québécoises, comme par ailleurs les Françaises, ne se départissent jamais d'une certaine méfiance lorsqu'elles invoquent les pionnières féministes américaines : elles considèrent que leurs problèmes ne sont pas tout à fait les mêmes et elles remarquent que, si forts que soient les textes américains, ils ne sont pas encore parvenus à faire advenir la révolution.

1. Amantes (Nicole Brossard, Amantes, éditions Quinze, Montréal, 1980) porte en exergue une citation de Mary Daly («it celebrates cerebral spinning », p. 9), quelques pages plus loin on trouve un extrait de poème d'Adrienne Rich (p. 13) ; puis, dix pages plus loin, elle cite « Adrienne et Gertrude s'étaient entendues sur le mot saisissante » (p. 23), très probablement Adrienne Rich.

2. Louky Bersianik, L’Euguélionne, roman tryptique, Montréal, Éditions La Presse Itée, 1976.

3. «Il n'y avait pas grand chose avant que j'écrive L'Euguélionne. Ce qui m'a donné le coup d'envoi, c'est Kate Millett : même pas son livre, mais un article dans Le Nouvel Observateur sur son livre, La Politique du mâle. » (Louky Bersianik, L'Écriture, c'est les cris. Entretiens avec France Théoret, Montréal, Éditions du remue-ménage, 2014, p. 21). Larticle en question se trouve dans Le Nouvel Observateur, Paris, n 334, 5 avril 1971.

4. Par exemple dans L'Euguélionne, lorsque dans la dernière partie elle évoque l'escroquerie de la prétendue libération sexuelle, référence à une partie de l'ouvrage La Politique du mâle. « Le sociologue se moque de vous quand il raconte que la libération sexuelle est arrivée. Rien n'est moins vrai puisque cette soi-disant révolution n'a libéré qu'une fraction de la population, fraction dont la majorité est masculine, comme il se doit. Très peu de vos pareilles y trouvent leur compte. La plupart d'entre vous, ou bien sont enchaînées au "foyer" avec des jeunes à garder, ou bien, travaillant à l'extérieur toute la journée, entreprennent en rentrant leur "seconde journée de travail". Elles ont bien le temps celles-là de "s'envoyer en l'air" ! » Louky Bersianik, L'Euguélionne, op. cit., p. 619-620).

5. C'est Love (Notes From the Second Year) qui est cité ; aux côtés de Françoise d'Eaubonne, Rollande Ballorain (Le Nouveau Féminisme américain), Virginia Woolf, Elizabeth Janeway.

6. Louky Bersianik, L’Euguélionne, op. cit., p. 30. 
A. Turbiau. Traversées québécoises en littérature féministe

Un certain rejet des États-Unis peut même parfois s'exprimer à travers des arguments féministes, par exemple chez Denise Boucher :

Cinq millions d'Américaines ont lu : La Femme totale", cuvre dégénérée d'une Pink Panther. À Noël 76, il n’y avait que ce livre, près des caisses, dans nos librairies.

Pendant que se diluait dans leur système de communication notre féminisme. La tapetterie de l'esprit reprenait le pouvoir. [...] La religion flambe à nouveau en nos murs ${ }^{2}$.

Denise Boucher critique ici l'influence américaine qui lui paraît être une force réactionnaire dangereuse pour les femmes québécoises : une invasion (« nos librairies ») et un potentiel retour aux temps de la Grande Noirceur (« La religion flambe à nouveau dans nos murs »). Et puis les États-uniennes négligent les Québécoises, ce qui les frustre parfois ; en mai 1978 par exemple les féministes québécoises participent au Third Annual Congress of Inter-American Women Writers, à l'université d'Ottawa ; elles en reviennent agacées car malgré leur nombre - elles étaient une cinquantaine dont vingt écrivaines - elles se sont senties ignorées par les États-uniennes, plus intéressées par les femmes latino-américaines que par leurs sœurs méconnues du nord ${ }^{3}$.

En fait les féministes québécoises entretiennent des rapports bien plus étroits avec les Françaises qu'avec leurs consœurs du monde anglophone, même s'ils ne sont pas non plus dénués de toute tension. Le monde anglophone a gardé une avance dans le développement du féminisme :

The 1960s and 1970s correspond to the emergence of a wide movement, aimed at integrating perpectives on race, class, and difference with gender analysis within feminist currents that used English as their primary language of communication ${ }^{4}$.

Mais dans les milieux québécois francophones, il a fallu attendre encore une vingtaine d'années pour que ces perspectives soient vraiment prises en compte. Miléna Santoro explique :

1. La Femme totale est un livre conservateur publié aux États-Unis en 1973 par Marabel Morgan.

2. Denise Boucher, Madeleine Gagnon, Retailles, Montréal, Éditions l'Étincelle, 1977, p. 47.

3. Voir Paule Gilbert Lewis, op. cit., p. 92-93.

4. Paula Ruth Gilbert, Miléna Santoro (dir.), Transatlantic Passages: Literary and Cultural Relations between Quebec and Francophone Europe, McGill-Queen's University Press, Montréal, 2010, p. 55] Ma traduction : « Les années 1960 et 1970 correspondent à l'émergence d'un large mouvement, attaché à intégrer aux analyses de genre des perspectives de race, de classe et de différence dans les courants féministes qui utilisaient l'anglais comme leur principale langue de communication. » 
A. Turbiau. Traversées québécoises en littérature féministe

Our hypothesis is that this disparity in the integration of perspectives on the intersectionality of oppressions is linked to the dominance of a discourse built on the universal woman within French feminism ${ }^{1}$.

Linfluence des Françaises sur les écrivaines québécoises a en effet été très grande, elles ont gardé longtemps une conception du féminisme très similaire ; mais, contrairement à ce qui se passe avec les États-uniennes, on peut parler dans ce cas de collaboration plus que d'influence, car les Québécoises ont aussi contribué à nourrir les débats français.

\section{Collaborations transatlantiques}

Au cours de la décennie 1970 plusieurs événements d'envergure marquent les collaborations entre Françaises et Québécoises. En 1975 se tient à Montréal la première rencontre internationale des écrivaines sur le thème «La Femme et le langage ». À la suite de leurs échanges là-bas, Hélène Cixous, Annie Leclerc, françaises, et Madeleine Gagnon, québécoise, décident d'écrire ensemble un essai sur le rapport des femmes à la littérature, publié en 1977 : La Venue à l'écriture ${ }^{2}$. Lannée suivante la revue française Sorcières publie un numéro spécialement organisé par un groupe d'écrivaines québécoises, intitulé La Jasette ${ }^{3}$; à cette revue collaborent par la suite Nicole Brossard dans les numéros 7 et $18^{4}$, Louise Cotnoir dans les numéros 19 et $21(1980)^{5}$ et Jovette Marchessault dans le numéro 20 (1980) ${ }^{6}$. Réciproquement, Hélène Cixous est présentée comme figure tutélaire du numéro 21 des Herbes rouges ${ }^{7}$, en tant qu'écrivaine représentative du nouveau mouvement de l'« écriture féminine ». Dans les livres français il n'arrive que ponctuellement que les Québécoises soient citées ${ }^{8}$; en revanche dans

1. Ibidem. Ma traduction : « Notre hypothèse est que cette disparité par rapport à l'intégration des perspectives sur l'intersectionnalité des oppressions est liée à la domination d'un discours construit sur la femme universelle au sein du féminisme français. »

2. Hélène Cixous, Madeleine Gagnon, Annie Leclerc, La Venue à l'écriture, Union générale d'édition, Paris, 1977.

3. Revue Sorcières, $n^{\circ}$ 14, La Jasette, Paris, Éditions Stock, 1978.

4. Nicole Brossard, «Si je jouis », Sorcières $\mathrm{n}^{\circ}$ 7, Stock, Paris, 1977, p. 46. Nicole Brossard, « Les traces du manifeste », Sorcières $n^{\circ} 18$, Stock, Paris, 1979, p. VIII.

5. Louise Cotnoir, «À cause des linges », Sorcières $n^{\circ}$ 19, Stock, Paris, 1980, p. 62. Louise Cotnoir, «Salope », Sorcières $n^{\circ} 20$, Stock, Paris, 1980, p. 83.

6. Jovette Marchessault, « Les Vaches de nuit », Sorcières n 20, Stock, Paris, 1980, p. 151.

7. Les Herbes rouges, $\mathrm{n}^{\circ}$ 21, Montréal, 1974.

8. Par exemple chez Françoise d'Eaubonne, «Encore un témoignage. Le dernier. Celui-là vient du pays de Maria Chapdelaine et des quintuplées, du catholicisme au pouvoir et des trappeurs de notre littérature enfantine ", dans Françoise d'Eaubonne, Le Féminisme ou la mort, Paris, Éditions Pierre Horay, 1974, p. 84. 
A. Turbiau. Traversées québécoises en littérature féministe

les livres du Québec on cite aussi souvent les Françaises que les Québécoises ${ }^{1}$. Là aussi les femmes voyagent : Madeleine Gagnon se rend en Belgique et en France au début de la décennie 1970 pour rendre visite notamment à Claire Lejeune, Christiane Rochefort et Annie Leclerc $^{2}$. Louky Bersianik en 1978 part en séjour en Europe ; dans l'autre sens, Hélène Cixous donne des cours au Québec en 1973-1974 et y accepte des entretiens — notamment un avec Nicole Brossard pour Le Devoir ${ }^{3}$, Marie Cardinal séjourne à plusieurs reprises au Québec entre 1961 et les années 1990, et elle y mène une partie de sa carrière littéraire ${ }^{4}$. Elles échangent leurs livres, même si c'est de manière asymétrique : les Québécoises ont accès à la quasi-totalité de la production féministe française, les Françaises ont accès à quelques livres québécois seulement - Retailles est apparemment celui qu'elles ont le plus lu, il est en tout cas le plus souvent cité.

Dans l'ensemble, les littératures féministes du Québec et de la France ont à peu près les mêmes caractéristiques : on y retrouve le même fond de revendications politiques (lutte pour l'avortement, la contraception, contre les violences domestiques, les stéréotypes sexistes...), le même vocabulaire pour les exprimer, les mêmes tendances vers l'écriture du corps et du flux, le même type de travail sur la langue. Comme le remarquait Miléna Santoro dans son livre sur les rapports entre le féminisme québécois et le féminisme français, c'est là une part très importante du travail de ces autrices qu'on aurait tort d'oublier :

their sense of connection with other women in the world, a conscious solidarity of purpose that defies borders and distance. [...] [a] common matrix of ideas and strategies which effectively fuse the two communities into one larger, if loosely knit, literary context ${ }^{5}$.

1. Suzanne Lamy, critique des écritures féminines, cite par exemple «les textes de Chantal Chawaf, de Jeanne Hyvrard, d'Hélène Cixous, de Monique Wittig, de Victoria Thérame, de Mara, de Marguerite Duras..., ici, de France Théoret, Yolande Villemaire, Monique LaRue, Nicole Brossard, Carole Massé, Louise Dupré, Madeleine Gagnon et de plusieurs autres », dans «Lautre lecture », Actes du Congrès de l'Association québécoise des professeurs de français, avril 1982 ; repris dans Quand je lis je m'invente, Montréal, Éditions de l'Hexagone, 1984, p. 20.

2. Voir Miléna Santoro, Mothers of Invention: Feminist Authors and Experimental Fiction in France and Quebec, McGill-Queen's University Press, Montréal, 2002, p. 10.

3. Hélène Cixous, «Une Romancière et critique sur le phallocentrisme », entretien avec Nicole Brossard, Le Devoir, 30 novembre 1974, p. 16. Voir aussi Santoro, op. cit., p. 156.

4. Voir Gilbert, Santoro, op. cit., p. 140.

5. Santoro, op. cit., p. 15. Ma traduction : «leur sentiment d'une connexion avec d'autres femmes du monde, une solidarité d'objectif consciente qui défie les frontières et la distance [...] une matrice commune d'idées et de stratégies qui fond pour de bon ces deux communautés dans un contexte littéraire plus large, quoique distendu ». 
Létude rapprochée des œuvres de ces autrices révèle néanmoins que certains des partages idéologiques ne se font pas tout à fait sur les mêmes lignes qu'en France. En France, il n'y a pas d'autrice féministe de renom qui mêle étroitement dans son travail revendications ouvertement féministes, proches d'un point de vue matérialiste ou radical, et réflexions sur un texte-corps, plus proches du mouvement de l'« écriture féminine ${ }^{1} »$; on en trouve en revanche au Québec dans les œuvres de Louky Bersianik, Madeleine Gagnon, France Théoret ou Nicole Brossard - si bien que cette combinaison devient même caractéristique de l'écriture féministe québécoise. Louky Bersianik arrive sur la scène littéraire en 1976, au moment où elle publie son Euguélionne; dans ce roman à la forme hybride, parodie des textes chrétiens et de la culture patriarcale en même temps qu'utopie révolutionnaire, le féminisme est fondé sur des revendications très concrètes. De nombreux passages fournissent par exemple du matériau pour revendiquer le droit à un avortement libre : une scène d'avortement clandestin particulièrement horrifique ${ }^{2}$, une scène d'insultes publiques faites à une avortée par une foule d'hommes en colère ${ }^{3}$, une scène qui rappelle le « folklore » des outils et produits utilisés par les femmes pour avorter quand l'avortement n'est pas légal. ${ }^{4}$ Le thème de l'exploitation domestique, pensé comme un phénomène économique, est également récurrent - on le constate par exemple dans ce dialogue avec un serveur de restaurant :

- Même logé, même nourri trois fois par jour, je ne ferais pas cela sans être payé. C'est très fatigant, vous savez. C'est fatigant de servir, pour les garçons de table. Et pour ceux qui préparent la nourriture, c'est aussi très fatigant. Quant aux plongeurs, je n'ai pas besoin de vous dire qu'ils sont crevés et écœurés à la fin de la journée. En plus de notre salaire, qui n'est pas très haut je vous l'accorde, nous avons les pourboires, heureusement. Il n'y a personne sur la terre qui consentirait à travailler pour des prunes.

- Si, dit Omicronne, votre femme!

1. À la rigueur ce serait chez Monique Wittig qu'on pourrait trouver un tel mélange des genres : dans Les Guérillères (1969) ou Le Corps lesbien (1973) par exemple, la dénonciation du patriarcat et de la culture hétéro-centrée se conjugue à la reconstruction littéraire d'une culture féminine et/ou lesbienne. Mais il est exclu d'associer Monique Wittig aux tenantes de l'écriture féminine parce que les œuvres que l'on vient de citer ont été publiées avant l'essor de l'écriture féminine, et parce que par la suite elle a toujours refusé d'y être associée. Voir là-dessus Monique Wittig, Le Chantier littéraire, Lyon, Presses Universitaires de Lyon, 2010, p. $122-123$.

2. Louky Bersianik, L'Euguélionne, op. cit., chapitre XXXIII « Le terrain de camping », § 310-312, p. 173-174.

3. Idem., chapitre LXVI «Lépouse classique », § 480-481, p. 274] Il s'agit d'une représentation théâtrale : une foule de comédiens mime le comportement des hommes anti-avortement.

4. «Lavortement clandestin ? Bof ! Du folklore ! Du folklore poétique ! Du folklore les aiguilles à tricoter, les produits à déboucher les toilettes, à nettoyer les parquets, du folklore, le ricin, le détergent, les cintres, les couteaux ! », Idem, chap. XXX, « La grossesse nommée désir », § 829, p. 478. 
- Elle, ce n'est pas pareil, elle est logée et nourrie ${ }^{5} .$.

La symétrie soulignée par l'italique marque l'incohérence d'une société qui ne donne pas la même valeur au travail selon que c'est un homme ou une femme qui s'en acquitte. Madeleine Gagnon, quant à elle, a été marxiste avant d'être féministe : son écriture est dès le départ empreinte du vocabulaire marxiste révolutionnaire que l'on retrouve dans les analyses dites matérialistes ou radicales. Même dans La Venue à l'écriture, alors que le thème est bien spécifiquement littéraire, elle mentionne la réalité physique de la condition des femmes, par exemple la fréquence des hystérectomies au Québec ${ }^{2}$ ou la peur du viol commune aux femmes $^{3}$; il s'agit pour elle, dans l'écriture comme sur le terrain militant, de nommer

La place de la guerre : soumission économique, politique, psychique, sexuelle. La femme objet. La femme dans l'image du mâle. La femme marchandise : ménagère, putain, battue, castrée, etc. etc. ${ }^{4}$

Chez Nicole Brossard la tendance radicale de la lutte féministe est peut-être plus discrète en termes de revendications concrètes. Mais malgré l'abstraction des notions qu'elle convoque, celles-ci renvoient bien à l'analyse radicale. Dans L'Amèr elle peut parler de «mère matrice et matérialiste », qui « réorganise sa matière : vie privée et politique ${ }^{5}$ », ou parler de la mère « patriarcale ${ }^{6}$ » en reprenant ainsi les mots-clefs du féminisme matérialiste. Il lui arrive aussi de faire des allusions particulièrement claires à des analyses matérialistes de la société patriarcale :

Le seul produit dont la mère dispose comme mode d'échange demeure son enfant. Sa seule entreprise : cinquante pour cent de la matière première (l'ovule), gestation (l'utérus comme outil, instrument), production (l'accouchement), mise en marché (sa force de travail : bras, jambes, stress). Mais elle ne peut échanger son produit ${ }^{7}$.

5. Idem, chap. LXXI « Les beaux yeux du patron », § 507, p. 297.

2. «Ici, en Amérique du Nord, c'est le pays des hystérectomies. Il faut vraiment se battre et tenir à son ventre, aimer son utérus, ses ovaires et même ses règles, pour ne pas subir la grande opération. », Madeleine Gagnon, «Mon corps dans l'écriture », La Venue à l'écriture, op. cit., p. 101.

3. Voir par exemple Madeleine Gagnon, Lueur, Montréal, VLB éditeur, p. 21, p. 48-49.

4. Madeleine Gagnon, Denise Boucher, op. cit., p. 111.

5. Nicole Brossard, L’Amèr, Montréal, Éditions Typo, [1977] 2013, p. 35.

6. Idem, p. 24.

7. Idem, p. 29. 
A. Turbiau. Traversées québécoises en littérature féministe

On peut constater le même phénomène chez France Théoret : le propos est d'ordinaire plutôt centré sur l'intime et l'écriture mais il arrive que le texte formule avec force des convictions féministes et touche alors à une tendance matérialiste d'inspiration marxiste. Par exemple dans la section «Plaidoyer pour un droit à l'existence des femmes ! » du livre Une voix pour Odile, elle parle des femmes qui toute leur vie, même en grave état de dépression, sont capables d'abattre leur travail de ménagère à cause des « paroles de l'autorité paternellemaritale [qui] superstructurent encore leurs actes ${ }^{1} »$. Mais, comme je l'annonçais plus haut, aussi présentes que soient les analyses de type matérialiste chez ces écrivaines, on trouve aussi chez elles - et surtout, en même temps, au sein des mêmes ouvrages- des réflexions qui les rapprochent plus du mouvement de l'«écriture féminine ». C'est très net lorsque, dans La Jasette, Marthe Blackburn explique « nous, nous traduisions droit à l'avortement dans notre langage de $\operatorname{corps}^{2} \gg$ : dans l'écriture des féministes québécoises, le matérialisme côtoie l'« écriture féminine » - et parfois son essentialisme latent. C'est ainsi que Louky Bersianik ouvre Le Pique-nique sur l’Acropole, dans une référence explicite à Luce Irigaray, citée juste avant :

Mon corps à corps perdu s'implique, simplement il produit. [...] Corps producteur de mots, de salive muette, de mue, de linge propre, de mucus, de faillites, de jouissance. Ici je tords le linge à la main. C'est-à-dire à deux mains. À la main qui s'écrit et cyprine entre les lignes, en torsade, qui caresse dans les plis ${ }^{3}$.

Dès l'orée du récit, Louky Bersianik pose ainsi qu'il y a un rapport entre l'écriture, le corps, la jouissance, les fluides corporels et les activités socialement construites comme féminines (laver le linge) ; du reste, comme chez Madeleine Gagnon et chez toutes les participantes de « l'écriture féminine » influencées par Luce Irigaray, l'écriture de Louky Bersianik est saturée de références à la psychanalyse. Dans L’Amèr de Nicole Brossard, l'écriture est aussi rapprochée de la caresse et du désir ${ }^{4}$, du corps et de ses fonctions biologiques ${ }^{5}$. Dans Une voix pour Odile, France Théoret compile des réflexions matérialistes sur la condition des femmes et des formulations très claires d'un projet d'écriture féminine ; affirmant ne pas croire une se-

1. France Théoret, Une voix pour Odile, éditions des Herbes rouges, Montréal, 1978 (je souligne).

2. Marthe Blackburn, «Les jasettes intérieures », Sorcières, n 14, p. 29.

3. Louky Bersianik, Le Pique-nique sur l'Acropole, Montréal, VLB éditeur, 1979, p. 15.

4. «Lécrire sera qu'un regard de femme se pose sur les autres, sur les choses. À produire son propre lieu de désir. [...] Pour ne pas flétrir de chaque caresse. Pour ne pas assener la caresse. », Nicole Brossard, L'Amèr, op. cit., p. 26.

5. «Mon corps encore d'instinct tend. À (celui de l'origine) se reproduire. (Textuellement) il n'en est pas question. En toutes lettres JE SUIS STÉRILE. » Nicole Brossard, L’Amèr, op. cit., p. 57. 
A. Turbiau. Traversées québécoises en littérature féministe

conde à une fiction qui porterait avant tout un projet politique ${ }^{1}$, elle fait de son projet poétique la recherche d'une écriture du flux :

je suis occupée par le flux, le passage, l'existence, le refoulé, l'impensé, la négativité, l'en-deçà du monde [...] Refus du linéaire, fendue en deux comme mon sexe, deux et plus, ni logique de la dualité, ni discours de l'ordre (intériorisé) [...] Je suis autre, éclatement, morceaux de journées, images du corps ou représentation toujours gardée présente. Je suis le manque 2 .

Dans cet extrait, comme chez Bersianik, l'allusion à Luce Irigaray est claire : elle reprend son vocabulaire et ses métaphores du « sexe qui n'en est pas $u^{3}{ }^{3}$ ». C'est peut-être chez Madeleine Gagnon que se trouve l'exemple le plus frappant du rapprochement entre matérialisme et «écriture féminine » : elle qui est l'une des féministes les plus marxistes de l'époque est aussi l'une des participantes les plus productives de ce mouvement littéraire. Lueur est tout entier un récit qui tente de prolonger la psychanalyse, de retrouver un langage pré-œdipien et un rapport originel à la mère perdue. Dans La Venue à l'écriture elle posait déjà une équivalence corps-texte et féminisme-écriture féminine :

Je veux [...] Couler comme du bon lait que je buvais ou que je donne. La libération des femmes, ça veut dire la parole du corps ${ }^{4}$.

Chez les Québécoises, il n'y a donc pas d'incompatibilité foncière entre démarche matérialiste et démarche «féminine »: les deux se complètent, souvent au sein des mêmes œuvres, ce qui n'est pas le cas — ou très rarement — chez les Françaises. Pourtant les Québécoises s'appuient prioritairement sur leurs travaux : du côté du matérialisme, elles lisent Françoise d'Eaubonne, Christine Delphy, Monique Wittig, elles connaissent les manifestations françaises du MLF et ses actrices principales, les imitent parfois ${ }^{5}$; en ce qui concerne l'écriture fé-

1. «Le discours politique-théorique-fictif est abus de langage. Le militant parle pour dire une chose à la fois et toute fiction politique est un leurre. [...] Il y a le discours militant et il y a la fiction. Ce sont là deux discours. [...] La fiction politique est molle, molle, molle... », France Théoret, « Cochonneries », op. cit., p. 71.

2. France Théoret, op. cit., p. 58.

3. Luce Irigaray, Ce sexe qui n'en est pas un, éditions de Minuit, Paris, 1977. Les premières formulations de l'idée du sexe multiple dont les lèvres se touchent remontent à Spéculum (éditions de Minuit, Paris, 1974) et au texte «Ce sexe qui n’en est pas un » publié la même année dans les Cahiers du Grif, n 5.

4. Madeleine Gagnon, « Mon corps dans l'écriture », La Venue à l'écriture, op. cit., p. 86-87.

5. Louise Turcotte le signale par exemple à propos du mouvement lesbien radical : le séparatisme lesbien québécois est directement inspiré de ce qui se passe à partir de juin 1980 en France, lorsque Monique Wittig et ses amies affirment que «les lesbiennes ne sont pas des femmes » et que le lesbianisme n'est pas inclus dans le féminisme. Voir Louise Turcotte, «Itinéraire d'un courant politique : le lesbianisme radical au Québec », dans Demczuk, Remiggi, op. cit., p. 363-393, p. 364-367. 
A. Turbiau. Traversées québécoises en littérature féministe

minine, elles lisent Hélène Cixous, Annie Leclerc, Luce Irigaray, dont les textes sont si souvent cités qu'ils le sont souvent par simples allusions ${ }^{1}$. Miléna Santoro remarque que la plupart des textes théoriques québécois, à l'exception notable de «Mon corps dans l'écriture » de Madeleine Gagnon, sont publiés après $1980^{2}$, et tous ils sont des

products more than initiators of exchanges within Quebec's feminist community, both borrow from and build upon works by their French and American counterparts, tackling many of the same ideas ${ }^{3}$.

Ainsi avant les années 1980, lorsque les Québécoises produisent des textes littéraires originaux, elles s'appuient surtout sur des éléments théoriques étrangers. Comment comprendre alors que malgré cette influence forte des Françaises sur les Québécoises on ne retrouve pas tout à fait les mêmes lignes de partage dans les littératures féministes des deux pays ? On peut faire l'hypothèse que cela tient à la distance géographique et affective qui sépare les différentes actrices du jeu théorique ; on sait qu'en France les tensions interpersonnelles entre femmes du mouvement influencent les prises de position théoriques et les antagonisent ${ }^{4}$, tandis qu'au Québec une plus grande souplesse est possible.

Outre ces rapports d'influence Québécoises et Françaises entretiennent parfois des relations de collaboration directe. Miléna Santoro signale, à propos des commentaires que Madeleine Gagnon a faits sur sa rencontre particulièrement enthousiaste avec Hélène Cixous et Annie Leclerc, que les relations entre Québécoises et Françaises, si elles ne sont pas tout à fait symétriques, ont néanmoins une importance dans la construction des contextes littéraires des deux pays :

1. Voir l'exemple mentionné plus haut ; voir aussi les premières lignes du Pique-nique sur l'Acropole: « Toutes touchantes et de n'être pas touchées. Toutes impliquées » — sont nécessairement une allusion à l'image de Luce Irigaray du «sexe qui n'en est pas un », où deux lèvres se touchent en permanence ; même phénomène pour Hélène Cixous voir par exemple ce passage de France Théoret, «Encore l'énorme rire de la Méduse monte du ventre au cerveau. », dans Une voix pour Odile, Montréal, Les Herbes rouges, 1978, p. 27.

2. Elle cite La Lettre aérienne de Nicole Brossard (1985), L'Échappée des discours de l'œil de Madeleine OuelletteMichalska (1981), Entre raison et déraison de France Théoret (1987), La Théorie, un dimanche, collectif (1988).

3. Santoro, op. cit., p. 19. Ma traduction : « produits plutôt qu'initiateurs des échanges qui ont lieu à l'intérieur de la communauté féministe du Québec, [qui] à la fois empruntent aux travaux de leurs homologues français et américains et construisent à partir d'eux, s'attaquant souvent aux mêmes idées ».

4. Voir les travaux d'Audrey Lasserre sur la question, notamment son travail de thèse Histoire d'une littérature en mouvement : textes, écrivaines et collectifs éditoriaux du MLF, 1970-1981, Paris III Sorbonne Nouvelle, Paris, 2014. 
A. Turbiau. Traversées québécoises en littérature féministe

While one could legitimately argue that French feminist thought had more influence on the work of Quebec women writers than the reverse, such remarks show that the degree of awareness in each community of the other's presence and production was not insignificant ${ }^{1}$.

Le fait est que les collaborations sont assez sporadiques et que les Québécoises lisent plus souvent les Françaises que l'inverse ; néanmoins certaines collaborations comme celle de La Venue à l'écriture révèlent l'intérêt réciproque que se portent les féministes des deux pays. Le numéro 14 de Sorcières, La Jasette — assez rarement cité dans la bibliographie franco-québécoise - est un autre exemple de collaboration aboutie. D'un côté, il y a un rapport que l'on pourrait dire de sororité : les Québécoises sont bien renseignées sur l'avancée du mouvement de libération des femmes en France et, lorsqu'elles s'adressent aux Françaises, elles prennent souvent soin d'expliquer ce que celles-ci pourraient mal comprendre. Par exemple, le texte «La jazzette ou le parler des négresses blanches d'Amérique ${ }^{2}$ » qui ouvre La Jasette explique en détails ce que signifie le mot «jasette » et comment on l'emploie au Québec, avant de passer à un propos plus franchement politique. À la fin du numéro, Michèle Jean publie à son tour un article qui récapitule pour les Françaises l'histoire du mouvement féministe québécois. Mais il arrive aussi que les Québécoises ressentent un décalage entre leurs préoccupations et celles des Françaises. C'est ce qu'exprime France Théoret à l'une de ses amies françaises, « Lyliane »: si c'est une Française qui parle d'« aphasie » féminine — il s'agit d'un thème extrêmement récurrent dans les œuvres féministes tant françaises que québécoises —, cela ne résonne pas de la même manière que si c'était une Québécoise qui parlait :

Lyliane, tu parles de quasi-aphasie et je l'entends avec mon oreille de québécoise. Française, tu me dis cela. Le dire et l'écouter aussi comporte une transgression car nous n'en finissons plus québécois de vivre dans un rapport paternaliste avec vous. Je l'ai entendu dans notre parole animée précisément par les effritements du quotidien et en même temps, replacé dans nos espaces différents ${ }^{3}$.

1. Santoro, op. cit., p. 15. Ma traduction : «Alors qu'on pourrait légitimement avancer l'idée que la pensée féministe française a eu plus d'influence sur le travail des écrivaines québécoises que l'inverse, de telles remarques montrent qu'à l'intérieur de chaque communauté le degré de conscience de la présence et de la production de l'autre n'était pas insignifiant ».

2. La Graph, «La jazzette ou le parler des négresses blanches d'Amérique », Sorcières,n ${ }^{\circ}$ 14, op. cit., p. 4-6. L'expression « négresses blanches » est une allusion à un ouvrage anticolonialiste québécois publié en 1968 par Pierre Vallières (Nègres blancs d'Amérique. Autobiographie précoce d'un « terroriste » québécois, Montréal, Parti pris, 1968).

3. France Théoret, op. cit., p. 52. 
A. Turbiau. Traversées québécoises en littérature féministe

Entre amies elles se comprennent, mais les mots n'ont pas le même sens pour chacune ; pour France Théoret, le silence imposé aux femmes ressemble à celui qu'elle vit aussi en tant que Québécoise parlant une langue qui est la sienne tout en étant celle de l'ancien colonisateur au comportement «paternaliste ». D'ailleurs, Une voix pour Odile tout entier joue sur cette ambiguïté : il y est sans cesse question de parler une langue dans une autre langue, d'être décentrée dans sa propre langue — s'agit-il du problème de la langue québécoise par rapport à la langue française, ou de celui de la langue des femmes par rapport à la langue patriarcale ? En réalité, France Théoret tisse les deux de manière inextricable. C'est un phénomène paradoxal que l'on retrouve chez beaucoup d'écrivaines féministes québécoises, comme l'explique Shirley Neuman :

For the female as for the colonial subject, the only recourse is to foreground one's difference from the dominant discourse while speaking within that discourse. "Je suis double sexe, double histoire », writes Madeleine Gagnon, possessed of « ma langue de mâle » in which she has lived and of "ma langue de femme » which she is in the process of affirming. [...] In Canada this double discursivity is sometimes encoded as bilinguism, as the perilous movement between two languages. Where English is the second language, learned with a perceived loss of the first, the acquisition is presented as a suppression of the "mothertongue" by a colonizing mastertongue ${ }^{1}$.

Quant à ce «paternalisme » parfois ressenti par les Québécoises, il est lié à l'asymétrie des réseaux d'édition faisant le lien entre le Québec et la France. Alors que les librairies québécoises sont régulièrement approvisionnées en livres français, il est rare de trouver des livres québécois aux rayons français. De là, les Françaises connaissent mal les Québécoises et peuvent donner l'impression de négliger leurs apports ; La Jasette naît d'ailleurs d'une plainte exprimée par «cette amie québécoise de passage à Paris qui regrettait que nous manifestions trop peu l'intérêt que nous portions au mouvement des femmes du Québec. »² On constate jus-

1. Shirley Neuman, «Importing difference », in Shirley Neuman, Smaro Kamboureli (dir.), A Mazing Space. Writing Canadian Women Writing, Longspoon Newest, Alberta, 1986, p. 392-403, p. 402] Ma traduction : «Pour la femme comme pour le sujet colonisé, la seule solution est de mettre en avant sa non-conformité au discours dominant tout en parlant du sein de ce discours. "Je suis double sexe, double histoire", écrit Madeleine Gagnon, possédée par "ma langue de mâle" dans laquelle elle a vécu et par "ma langue de femme" qu'elle est en train d'affirmer. [...] Au Canada cette double discursivité est parfois codée comme un bilinguisme, comme le périlleux mouvement entre deux langages. Là où l'anglais est la seconde langue, apprise avec l'impression de perdre la première, son acquisition est présentée comme une répression de la "langue maternelle" par la langue colonisante du maître ».

2. Voir aussi le texte de Claire de Lamirande, «Les écrivains d'ici ont-ils des droits ? », dans Sorfleet, op. cit., p. 79-88. 
A. Turbiau. Traversées québécoises en littérature féministe

tement dans cet éditorial que le discours des Françaises est parfois ambigu ; il adopte en l'occurrence un ton effectivement un peu «paternaliste », c'est-à-dire dénotant en même temps bienveillance et distance. Léditorial commence ainsi par marquer un éloignement :

Il nous faudrait sans aucun doute débattre entre nous, lectrices et/ou collaboratrices de Sorcières, de ce sentiment d'exclusion vécu douloureusement par certaines d'entre-nous à l'égard d'une revue dont se circonscriraient ainsi les limites : revue parisienne écrite et fabriquée par des femmes vivant à Paris ${ }^{1}$.

D’abord le déterminant « certaines » détache les rédactrices de l'éditorial de celles qui se plaignent ; puis l'usage répété du conditionnel ancre le propos dans le registre de l'hypothèse, de l'idée intéressante à évaluer mais non véridique ; juste après, l'éditorial signale que la revue a déjà par le passé fait la preuve qu'elle était plus ouverte que ce que les critiques laissent entendre - en effet, il est arrivé une fois que des Alsaciennes prennent la direction de la revue ${ }^{2}$. La critique des Québécoises est ainsi prise en compte par la revue sans être assumée jusqu'au bout. Plus loin, on peut constater que leur projet est présenté de manière quelque peu exotique : l'accent est porté sur la distance qui éloigne les deux pays («malgré l'Océan », « courrier si long », «éloignement géographique impressionnant », «c'est loin le Québec !») — soulignée pour pouvoir y opposer rhétoriquement la proximité de pensée qui caractérise les deux pays. Laccent est également porté sur la surprise qu'ont ressentie les Françaises :

Que vous ayez ressenti le besoin de publier un numéro québécois, ici à Paris, alors qu'il se passe tant de choses chez vous, nous a paru étonnant mais intéressant [...] une ouverture certainement très riche. [...] Nous souhaitons que amour et attention, portés à vous québécoises, présideront à la lecture, par nous françaises, de ces pages qui sont les vôtres ${ }^{3}$.

Les Françaises apparaissent perplexes, curieuses ; les termes choisis — «intéressant », « certainement très riche » - signalent qu'elles ne savent pas à quoi s'attendre, qu'elles n'ont que vaguement idée de ce à quoi ressemble la production québécoise. La distance continue d'être marquée par l'opposition répétée entre «vous » et «nous » et par l'incise « ici à Paris » placée en concurrence de « chez vous ». La distinction est révélatrice, car un « nous » sororal aurait pu être choisi - dans le compte rendu du livre Retailles qui est fait en fin de numéro,

1. «Éditorial », Sorcières, $n^{\circ} 14$, op. cit. Pas de numéro de page indiqué.

2. Ibidem.

3. Ibid. 
A. Turbiau. Traversées québécoises en littérature féministe

c'est ce «nous » qui est utilisé. Il semble donc qu'à l'orée de la revue, les différences nationales soient considérées comme plus intéressantes que la proximité idéologique. Le même mélange de curiosité bienveillante et de plaisir distancié se retrouve, ailleurs, sous la plume d'autres féministes françaises ; citons pour exemple le compte rendu que Christiane Rochefort fait de Retailles:

En voici deux qui osent. [...]

Et en quelle langue! En français. Un français qui n’a pas été asservi par le Pouvoir, dressé poli, taillé émondé, raboté laminé aplati. Ne parle pas de haut, ne pisse pas froid. Enfin, du français! Quel plaisir, quelle leçon, et quel bain!

Des poètes de la poésie faite par tous. Des femmes. Des Québécoises.

Léloge porte avant tout sur le propos politique de l'ouvrage : Christiane Rochefort félicite Madeleine Gagnon et Denise Boucher d'avoir su si bien dire les problèmes internes des groupes féministes ; mais pour louer la valeur proprement littéraire du texte, elle passe par l'exotisation de la langue québécoise - langue française à l'histoire autre - dont elle se distancie. Le mot final est sans doute significatif : pourquoi dire «Québécoises 》 après «Femmes », après «tous »? Le mouvement va du plus général au plus particulier : Rochefort signale aux Françaises que si elles doivent aller lire ce livre, c'est aussi justement parce qu'il ne peut pas vraiment ressembler aux leurs. Ainsi, les Françaises parlant des Québécoises ou travaillant avec elles ont tendance à accentuer la distance qui les sépare, tandis qu'en général, dans les textes québécois, la proximité ressentie avec les Françaises n'est pas vraiment un enjeu, Françaises et Québécoises étant citées de la même manière.

\section{Conclusion}

La littérature féministe québécoise est ainsi, pendant les années 1970, portée par des échanges internationaux. Les Québécoises se nourrissent de la pensée et des nouveaux codes culturels venus des États-Unis ; elles y voyagent, elles transmettent ce qu'elles y apprennent à leurs sœurs canadiennes. En revanche, il n’y a pas de réelle réciprocité à ces échanges, ou celle-ci est trop ponctuelle pour pouvoir signifier une vraie collaboration entre les femmes des deux pays. Ce sont surtout les militantes et théoriciennes féministes qui intéressent les Québé-

1. Citation tirée d'un texte dactylographié conservé dans les archives de Christiane Rochefort de l'IMEC, cote RCF 37.3, intitulé « Pour Retailles» - reproduit ici avec l'accord de l'IMEC. À ce jour je ne sais pas encore où l'article a été publié. 
A. Turbiau. Traversées québécoises en littérature féministe

coises : Kate Millett, Shulamith Firestone, Betty Friedan, même quand elles sont aussi autrices de littérature, sont surtout citées pour leurs essais militants - la seule exception concerne les écrivaines lesbiennes féministes (Djuna Barnes, Adrienne Rich, Mary Daly), souvent à la fois essayistes et romancières ou poètes, et régulièrement citées par Nicole Brossard. Avec les Françaises, les rapports sont différents : les Québécoises les connaissent souvent mieux que les États-uniennes et tentent de véritables échanges avec elles pour construire ensemble une littérature révolutionnaire. Des Françaises, elles glanent tous types de travaux : elles s'intéressent aux essais historiques et féministes de Françoise d'Eaubonne, aux essais psychanalytiques de Luce Irigaray plus encore, à la nouvelle littérature de l'« écriture féminine » principalement portée par Hélène Cixous et Annie Leclerc, aux revues de femmes auxquelles elles ont accès outre-Atlantique. Quelques échanges ont lieu, dont l'initiative revient en général aux Québécoises : ces femmes se rendent visite, écrivent livres et revues ensemble, essaient tant que faire se peut de lire les productions des unes et des autres.

Au moment des années 1970, la littérature féministe québécoise se construit de manière indépendante, élaborant matières et formes de manière tout à fait originale et différente de ce que l'on peut lire au même moment en France ou aux États-Unis, s'avérant même parfois pionnière en matière de rapport à la langue ${ }^{1}$. Toutefois, elle tire très largement son inspiration des théories féministes et littéraires qui sont en train de se développer dans les «centres » hégémoniques que sont les États-Unis et la France. À partir des années 1980, comme l'ont étudié Suzanne Lamy, Louise Dupré, Lori Saint-Martin ou encore Isabelle Boisclair $^{2}$, la tendance changera : les Québécoises produiront leurs propres outils théoriques et critiques et recentreront leurs analyses sur les spécificités de leurs propres créations, tout en restant en dialogue ouvert avec les productions des autres pays. C'est pourquoi l'on peut parler de «traversées québécoises », selon le titre que j'ai souhaité donner à cet article et que j'ai expliqué en introduction : la littérature féministe québécoise ne s'est jamais placée en situation de dépendance par rapport aux créations états-uniennes et françaises, elle les a plutôt visitées pour en tirer de quoi se construire elle-même, avec ou sans leur participation active.

1. Louky Bersianik est apparemment la première personne du monde francophone à avoir proposé une analyse linguistique approfondie de la féminisation des noms de métier. Voir L'Écriture, c'est les cris, op. cit., «La féminisation », p. 93-112.

2. Voir Suzanne Lamy, Quand je lis je m’invente, Montréal, Éditions de l'Hexagone, 1984. Louise Dupré, Stratégies du vertige. Trois poètes : Nicole Brossard, Madeleine Gagnon, France Théoret, Montréal, Éditions du remueménage, 1989. Lori Saint-Martin, Contre-voix. Essais de critique au féminin, Montréal, Nuit Blanche, 1997. Isabelle Boisclair, Ouvrir la voie/x. Le processus constitutif d'un sous-champ littéraire féministe au Québec (19601990), Montréal, Éditions Nota Bene, 2004. 